\title{
The Lattice Structure of Connection Preserving Deformations for $q$-Painlevé Equations I
}

Christopher M. ORMEROD

La Trobe University, Department of Mathematics and Statistics, Bundoora VIC 3086, Australia E-mail: C.Ormerod@latrobe.edu.au

Received November 26, 2010, in final form May 03, 2011; Published online May 07, 2011

doi:10.3842/SIGMA.2011.045

\begin{abstract}
We wish to explore a link between the Lax integrability of the $q$-Painlevé equations and the symmetries of the $q$-Painlevé equations. We shall demonstrate that the connection preserving deformations that give rise to the $q$-Painlevé equations may be thought of as elements of the groups of Schlesinger transformations of their associated linear problems. These groups admit a very natural lattice structure. Each Schlesinger transformation induces a Bäcklund transformation of the $q$-Painlevé equation. Each translational Bäcklund transformation may be lifted to the level of the associated linear problem, effectively showing that each translational Bäcklund transformation admits a Lax pair. We will demonstrate this framework for the $q$-Painlevé equations up to and including $q-\mathrm{P}_{\mathrm{VI}}$.
\end{abstract}

Key words: $q$-Painlevé; Lax pairs; $q$-Schlesinger transformations; connection; isomonodromy

2010 Mathematics Subject Classification: 34M55; 39A13

\section{Introduction and outline}

The discrete Painlevé equations are second order non-linear non-autonomous difference equations admitting the the Painlevé equations as continuum limits [22]. They are considered integrable by the integrability criterion of singularity confinement [22], solvability via associated linear problems [21] and have zero algebraic entropy [2]. There are three classes of discrete Painlevé equations: the additive difference equations, $q$-difference equations and elliptic difference equations. This classification is in accordance with the way in which the nonautonomous variable evolves with each application of the iterative scheme. The aim of this article is to derive a range of symmetries of the considered $q$-difference Painlevé equations using the framework of their associated linear problems.

The discovery of the solvability of a $q$-Painlevé equation via an associated linear problem was established by Papageorgiou et al. [21]. It was found that a $q$-Painlevé equation can be equivalent to the compatibility condition between two systems of linear $q$-difference equations, given by

$$
\begin{aligned}
& Y(q x, t)=A(x, t) Y(x, t), \\
& Y(x, q t)=B(x, t) Y(x, t),
\end{aligned}
$$

where $A(x, t)$ and $B(x, t)$ are rational in $x$, but not necessarily $t$, which is the independent variable in the $q$-Painlevé equation. A difference analogue of the theory of monodromy preserving deformations [13, 11, 12] was proposed by Jimbo and Sakai [14], who introduced the concept of connection preserving deformations for $q$-Painlevé equations. Given a system of linear $q$ difference equations, of the form (1.1a), conditions on the existence of the formal solutions and connection matrix associated with (1.1a) has been the subject of Birkhoff theory $[4,6,1]$. The preservation of this connection matrix leads naturally to the auxiliary linear problem in $t$, given 
by (1.1b). This theory also led to the discovery of the $q$-analogue the sixth Painlevé equation in an analogous manner to the classical result of Fuchs $[9,8]$.

There was one unresolved issue in the explanation of the emergence of discrete Painlevé type systems, and this was related to the emergence, and definition, of $B(x, t)$. Unlike the theory of monodromy preserving deformations and the surfaces of initial conditions where there exists just one canonical Hamiltonian flow [27, 13], there are many ways to preserve the connection matrix. According to the existing framework, one may determine $B(x, t)$ from a known change in the discrete analogue of monodromy data, i.e., the characteristic data. However, we would argue that this known change is not canonical, or unique. That is to say that by considering all possible changes in the characteristic data, one formulates a system of Schlesinger transformations inducing a system of Bäcklund transformations of the Painlevé equation. A particular case of this theory was studied by the author in connection with the $q$-difference equation satisfied by the Big $q$-Laguerre polynomials [20].

This concept is not entirely new, one only needs to examine Jimbo and Miwa's second paper [11] to see a set of discrete transformations, known as Schlesinger transformations, specified for the Painlevé equations. These Schlesinger transformations can be thought of as a discrete group of monodromy preserving evolutions, in which various elements of the monodromy data are shifted by integer amounts. The compatibility between the discrete evolution and the continuous flow induces a Bäcklund transformation of the Painlevé equation, which appear in the associated linear problem, and in some cases, induce the evolution of some discrete Painlevé equations of additive type [15].

We will show the same type of transformations for systems of $q$-difference equations describe similar transformations. We consider systems of transformations in which (1.1a) is quadratic. There are only a finite number of cases in which $A(x)$ is quadratic, and these cases cover the $q$-Painlevé equations up to and including the $q$-analogue of the sixth Painlevé equation [14]. We will consider the set of transformations of the associated linear problems for the exceptional $q$-Painlevé equations in a separate article as we have preliminary results, including a Lax pair for one of these equations that seems distinct from those that have appeared in [31] and [25].

These transformations may be derived in an analogous manner to the differential case. If one knows formal expansions of the solutions of (1.1a) at $x=0$ and $x=\infty$, then one may formulate the Schlesinger transformations directly in terms of the expansions. Formal expansions for regular $q$-difference equations are well established in the integrable community $[4,14]$, however, many associated linear problems for $q$-Painlevé equations fail to be regular at 0 or $\infty$. Hence, it is another goal of this article to apply the more general expansions provided by Adams [1] and Guenther [5] to derive the required Schlesinger equations. These expansions seem to be not as well established, however, all known examples of connection preserving deformations seem to fit nicely a framework that encompasses the regular and irregular cases [14, 17, 21]. In previous studies, such as those by by Sakai $[24,14], B(x, t)$ from $(1.1 b)$ and the Painlevé equations seem to have been derived from an overdetermined set of compatibility conditions. In an analogous manner to the continuous case, the formal solutions obtained may or may not converge in any given region of the complex plane [28].

The most important distinction we wish to make here is the absence of a variable $t$. We remark that this should be seen as a consistent trend in the studies of discrete Painlevé equations, whereby the framework of Sakai [23] and the work of Noumi et al. [18] show us that we should consider the Bäcklund transformations and discrete Painlevé equations on the same footing.

The outline of this article is as follows: In Section 2 we introduce some special functions associated with $q$-linear systems. In Section 3 we shall outline the main results of Birkhoff theory as presented by Adams and Guenther [1,5] and subsequently, specify the natural lattice structure of the connection preserving deformations. In Section 4 we will explore the quadratic matrix polynomial case in depth. Even at this level, we uncover a series of Lax-pairs. 


\section{$2 \quad q$-special functions}

A general theory of special $q$-difference equations, with particular interest in basic hypergeometric series, may be found in the book by Gasper and Rahman [10]. The work of Sauloy and Ramis provide some insight as to how these $q$-special functions both relate to the special solutions of the linear problems and the connection matrix [26,7], as does the work of van der Put and Singer [29, 30]. For the following theory, it is convenient to fix a $q \in \mathbb{C}$ such that $|q|<1$, in order to have given functions define analytic functions around 0 or $\infty$.

We start by defining the $q$-Pochhammer symbol [10], given by

$$
(a, q)_{k}= \begin{cases}\prod_{n=1}^{k}\left(1-a q^{n-1}\right) & \text { for } k \in \mathbb{N} \\ \prod_{n=1}^{\infty}\left(1-a q^{n-1}\right) & \text { for } k=\infty \\ 1 & \text { for } k=0\end{cases}
$$

We note that $(a, q)_{\infty}$ satisfies

$$
(q x ; q)_{\infty}=\frac{1}{1-x}(x ; q)_{\infty},
$$

or equivalently,

$$
\left(\frac{x}{q} ; q\right)_{\infty}=\left(1-\frac{x}{q}\right)(x ; q)_{\infty}
$$

We define a fundamental building block; the Jacobi theta function [10], given by the biinfinite expansion

$$
\theta_{q}(x)=\sum_{n \in \mathbb{Z}} q^{\left(\begin{array}{c}
n \\
2
\end{array}\right)} x^{n}
$$

which satisfies the $q$-difference equation

$$
x \theta_{q}(q x)=\theta_{q}(x) .
$$

The second function we require to describe the asymptotics of the solutions of (1.1a) is the $q$-character, given by

$$
e_{q, c}(x)=\frac{\theta_{q}(x) \theta_{q}(c)}{\theta_{q}(x c)}
$$

which satisfies

$$
e_{q, c}(q x)=c e_{q, c}(x), \quad e_{q, q c}(x)=x e_{q, c}(x) .
$$

Using the above functions, we are able to solve any one dimensional problem.

\section{Connection preserving deformations}

We will take a deeper look into systems of linear $q$-difference equations of the form

$$
y\left(q^{n} x\right)+a_{n-1}(x) y\left(q^{n-1} x\right)+\cdots+a_{1}(x) y(q x)+a_{0}(x) y(x)=0,
$$


where the $a_{i}(x)$ are rational functions. One may easily express a system of this form as a matrix equation of the form

$$
Y(q x)=A(x) Y(x)
$$

where $A(x)$ is some rational matrix. We quote the theorem of Adams [1] suitably translated for matrix equations by Birkhoff [5] in the revised language of Sauloy et al. [26, 29].

Theorem 3.1. Under general conditions, the system possesses formal solutions given by

$$
Y_{0}(x)=\hat{Y}_{0}(x) D_{0}(x), \quad Y_{\infty}(x)=\hat{Y}_{\infty}(x) D_{\infty}(x),
$$

where $\hat{Y}_{0}(x)$ and $\hat{Y}_{\infty}(x)$ are series expansions in $x$ around $x=0$ and $x=\infty$ respectively and

$$
D_{0}(x)=\operatorname{diag}\left(\frac{e_{q, \lambda_{i}}(x)}{\theta_{q}(x)^{\nu_{i}}}\right), \quad D_{\infty}(x)=\operatorname{diag}\left(\frac{e_{q, \kappa_{i}}(x)}{\theta_{q}(x)^{\nu_{i}}}\right) .
$$

Given the existence and convergence of these solutions, we may form the connection matrix, similar to that of difference equations [3], given by

$$
P(x)=Y_{\infty}(x) Y_{0}(x)^{-1} .
$$

In a similar manner to monodromy preserving deformations, we identify a discrete set of characteristic data, namely

$$
M=\left\{\begin{array}{cccccc}
\kappa_{1} & \ldots & \kappa_{n} & & & \\
\lambda_{1} & \ldots & \lambda_{n} & a_{1} & \ldots & a_{m}
\end{array}\right\} .
$$

Defining this characteristic data may not uniquely define $A(x)$ in general. In the problems we consider, which are also those problems considered previously in the work of Sakai [14], Murata [17] and Yamada [31], the characteristic data defines a three dimensional linear algebraic group as a system of two first order linear $q$-difference equations, but is two dimensional as one second order $q$-difference equation. The gauge freedom disappears when one extracts the second order $q$-difference equation from the system of two first order $q$-difference equations. Let us suppose the linear algebraic group is of dimension $d$, then let us introduce variables $y_{1}, y_{2}, \ldots$, $y_{d}$, which parameterize the linear algebraic group. We may now set a co-ordinate system for this linear algebraic group, hence, we write

$$
M_{A}=\left\{\begin{array}{lllllll}
\kappa_{1} & \ldots & \kappa_{n} & & \\
\lambda_{1} & \ldots & \lambda_{n} & a_{1} & \ldots & a_{m} & : y_{1}, \ldots, y_{d}
\end{array}\right\}
$$

as defining a matrix $A$.

By considering the determinant of the left hand side of (1.1a) and the fundamental solutions specified by (3.1), one is able to show

$$
\prod_{i=1}^{n} \kappa_{i} \prod_{j=1}^{m}\left(-a_{j}\right)=\prod_{i=1}^{n} \lambda_{i}
$$

which forms a constraint on the characteristic data.

We now explore connection preserving deformations [14]. Let $R(x)$ be a rational matrix in $x$, then we apply a transformation $Y \rightarrow \tilde{Y}$ by the matrix equation

$$
\tilde{Y}(x)=R(x) Y(x) .
$$


The matrix $\tilde{Y}$ satisfies a matrix equation of the form (1.1a), given by

$$
\tilde{Y}(q x)=\left[R(q x) A(x) R(x)^{-1}\right] \tilde{Y}(x)=\tilde{A}(x) \tilde{Y}(x) .
$$

We note that if $R(x)$ is rational and invertible, then

$$
\begin{aligned}
\tilde{P}(x) & =\left(\tilde{Y}_{\infty}(x)\right)^{-1} \tilde{Y}_{0}(x)=\left(R(x) Y_{\infty}(x)\right)^{-1} R(x) Y_{0}(x) \\
& =\left(Y_{\infty}(x)\right)^{-1} R(x)^{-1} R(x) Y_{0}(x)=Y_{\infty}(x) Y_{0}(x),
\end{aligned}
$$

hence, the system defined by

$$
\tilde{Y}(q x)=\tilde{A}(x) \tilde{Y}(x),
$$

is a system of linear $q$-difference equations that possesses the same connection matrix. However, the left action of $R(x)$ may have the following effects: The transformation may

- change the asymptotic behavior of the fundamental solutions at $x=\infty$ by letting $\kappa_{i} \rightarrow$ $q^{n} \kappa_{i}$

- change the asymptotic behavior of the fundamental solutions at $x=0$ by letting $\lambda_{i} \rightarrow q^{n} \lambda_{i}$;

- change the position of a root of the determinant by letting $a_{i} \rightarrow q^{n} a_{i}$.

We use the same co-ordinate system for $\tilde{A}(x)$ as we did for $A(x)$ via (3.2), giving

$$
M_{\tilde{A}}=\left\{\begin{array}{cccccc}
\tilde{\kappa}_{1} & \ldots & \tilde{\kappa}_{n} & & \\
\tilde{\lambda}_{1} & \ldots & \tilde{\lambda}_{n} & \tilde{a}_{1} & \ldots & \tilde{a}_{m}
\end{array}: \tilde{y}_{1}, \ldots, \tilde{y}_{d}\right\} .
$$

Naturally, $R(x)$ is a function of the characteristic data and the $y_{i}$, hence, we write

$$
R(x)=\tilde{Y}_{0}(x) Y_{0}(x)^{-1}=\tilde{Y}_{\infty}(x) Y_{\infty}(x)^{-1}=R\left(x ; y_{i}, \tilde{y}_{i}, \kappa_{j}, \tilde{\kappa}_{j}, \tilde{\lambda}_{l}, \lambda_{l}, a_{n}, \tilde{a}_{n}\right) .
$$

An alternate characterization of (3.5) is the compatibility condition resulting the consistency of (3.4) with (1.1a), which gives

$$
\tilde{A}(x) R(x)=R(q x) A(x),
$$

which has appeared many times in the literature [14, 21, 24]. Given a suitable parameterization, we obtain a rational map on the co-ordinate system for $A(x)$ :

$$
T: M_{A} \rightarrow M_{\tilde{A}},
$$

For each $i=1, \ldots, d$, we use (3.6) to find a rational mapping $\phi_{i}$ such that

$$
\tilde{y}_{i}=\phi_{i}\left(M_{A}\right) \text {, }
$$

where $M_{A}$ includes all of the variables in (3.2).

We now draw further correspondence with the framework of Jimbo and Miwa [11]. Let $\left\{\mu_{1}, \ldots, \mu_{K}\right\}$ be a collection of elements in the characteristic data, and $\left\{m_{1}, \ldots, m_{K}\right\}$ be a set of integers, then we define the transformation

$$
T_{\mu_{1}^{m_{1}}, \ldots, \mu_{K}^{m_{K}}}: M_{A} \rightarrow M_{\tilde{A}}
$$

to be the transformation that multiplies $\mu_{i}$ by $q^{m_{i}}$ leaving all other characteristic data fixed. The $m_{i}$ have to be chosen so that $M_{\tilde{A}}$ satisfies (3.3). The group of Schlesinger transformations, $G_{A}$, is the set of these transformation with the operation of composition

$$
G_{A}=\left\langle T_{\mu_{1}^{m_{1}}, \ldots, \mu_{K}^{m_{k}}, \circ},\right\rangle
$$


This group is generated by a set of elementary Schlesinger transformations which only alter two variables, i.e., $K=2$. For example, if wish to multiply $\kappa_{1}$ by $q$ and $a_{1}$ by $q^{-1}$, so to preserve (3.3), this would be the elementary transformation $T_{\kappa_{1}, a_{1}^{-1}}$. Since the subscripts define the change in characteristic data, we need only specify the relation between the $y_{i}$ and $\tilde{y}_{i}$, hence, we will write

$$
T_{\mu_{1}^{m_{1}}, \ldots, \mu_{K}^{m_{K}}}: \tilde{y}_{1}=\phi_{1}\left(M_{A}\right), \quad \ldots, \quad \tilde{y}_{d}=\phi_{d}\left(M_{A}\right) .
$$

We will denote matrix that induces the transformation $T_{\mu_{1}^{m_{1}}, \ldots, \mu_{K}^{m_{K}}}$ by $R_{\mu_{1}^{m_{1}}, \ldots, \mu_{K}^{m_{K}}}$ so that the transformation $M_{A} \rightarrow M_{\tilde{A}}$ is specified by the transformation of the linear problem given by

$$
\tilde{Y}(x)=R_{\mu_{1}^{m_{1}}, \ldots, \mu_{K}^{m_{K}}}(x) Y(x) .
$$

The goal of the the later sections will be to construct a set of elementary Schlesinger transformations that generate $G_{A}$ and describe the corresponding $R$ matrices.

\section{$4 \quad q$-Painlevé equations}

We now look at the group of Schlesinger transformations for the $q$-Painlevé equations. In accordance with the classification of Sakai [23], we have ten surfaces considered to be of multiplicative type. Each surface is associated with a root subsystem of a root system of type $E_{8}^{(1)}$. We may label the surface in two different ways; either the type of the root system of $E_{8}^{(1)}, R$, or the type of the root subsystem of the orthogonal complement of $R, R^{\perp}$. Given a surface of initial conditions associated with a root subsystem of type $R$, the Painlevé equation itself admits a representation of an affine Weyl group of type $R^{\perp}$ as a group of Bäcklund transformations. The degeneration diagram for these surfaces is shown in Fig. 1 where we list both $R$ along with $R^{\perp}$.

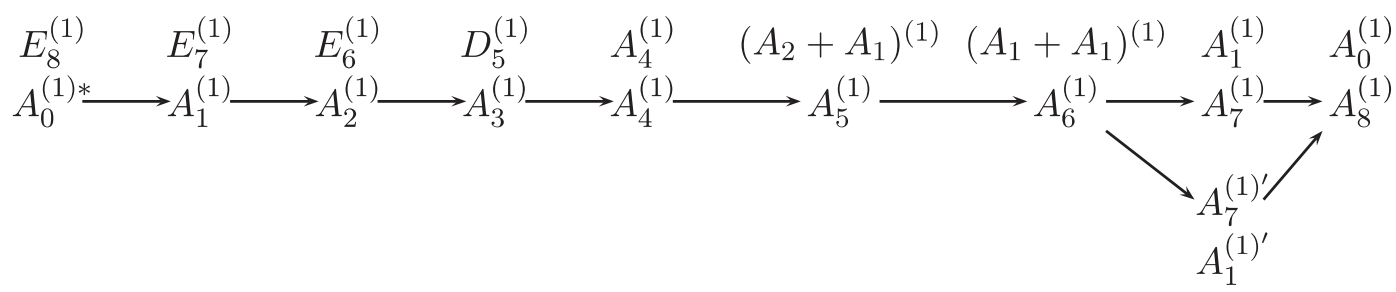

Figure 1. This diagram represents the degeneration diagram for the surfaces of initial conditions of multiplicative type [23].

In this article we will discuss the group of connection preserving deformations for $q$-Painlevé equations for cases up to and including the $A_{3}^{(1)}$. As the $A_{8}^{(1)}$ surface does not correspond to any $q$-Painlevé equation, this gives us six cases to consider here. These are also the set of cases in which $A(x)$ is quadratic in $x$. We will consider the higher degree cases, which include associated linear problem for the Painlevé equations whose Bäcklund transformations represent the exceptional affine Weyl groups in a separate article.

It has been well established that the degree one case of (1.1a), where $A(x)=A_{0}+A_{1} x$ is completely solvable in general in terms of hypergeometric functions [16]. It is also interesting to note that the basic hypergeometric difference equation fits into the framework of connection preserving deformations. The result of the above transformation group reproduces many known transformations [10]. 
Table 1. The correspondence between the data that defines the linear problem and the $q$-Painlevé equations. This data includes the determinant, and the asymptotic behavior of the solutions at $x=0$ and $x=\infty$ in $(3.1)$.

\begin{tabular}{|c|c|c|c|c|}
\hline & $\operatorname{det} A$ & $\left(\mu_{1}, \mu_{2}\right)$ & $\left(\nu_{1}, \nu_{2}\right)$ & $\operatorname{dim} G_{A}$ \\
\hline \hline $\mathrm{P}\left(A_{7}^{(1)}\right)$ & $\kappa_{1} \kappa_{2} x^{3}$ & $(2,1)$ & $(0,3)$ & 3 \\
$\mathrm{P}\left(A_{7}^{(1)^{\prime}}\right)$ & $\kappa_{1} \kappa_{2} x^{2}$ & $(2,0)$ & $(0,2)$ & 3 \\
$\mathrm{P}\left(A_{6}^{(1)}\right)$ & $\kappa_{1} \kappa_{2} x^{2}\left(x-a_{1}\right)$ & $(2,1)$ & $(0,2)$ & 4 \\
$\mathrm{P}\left(A_{5}^{(1)}\right)$ & $\kappa_{1} \kappa_{2} x\left(x-a_{1}\right)\left(x-a_{2}\right)$ & $(2,1)$ & $(0,1)$ & 5 \\
$\mathrm{P}\left(A_{4}^{(1)}\right)$ & $\kappa_{1} \kappa_{2}\left(x-a_{1}\right)\left(x-a_{2}\right)\left(x-a_{3}\right)$ & $(2,1)$ & $(0,0)$ & 6 \\
$\mathrm{P}\left(A_{3}^{(1)}\right)$ & $\kappa_{1} \kappa_{2}\left(x-a_{1}\right)\left(x-a_{2}\right)\left(x-a_{3}\right)\left(x-a_{4}\right)$ & $(2,2)$ & $(0,0)$ & 7 \\
\hline
\end{tabular}

\section{$4.1 \quad q-\mathrm{P}\left(A_{7}^{(1)}\right)$}

Let us consider the simplest case in a more precise manner than the others as a test case. The simplest $q$-difference case is the

$$
\left\{b_{0}, b_{1}: f, g\right\} \rightarrow\left\{\frac{b_{0}}{q}, b_{1} q: \tilde{f}, \tilde{g}\right\}
$$

where $q=b_{0} b_{1}$ and the evolution is

$$
\tilde{f} f=\frac{b_{1}(1-\tilde{g})}{\tilde{g}}, \quad \tilde{g} g=\frac{1}{f},
$$

which is also known as $q-\mathrm{P}_{\mathrm{I}}$. We note that this transformation gives us a copy of $\mathbb{Z}$ inside the group of Bäcklund transformations [23]. We note that there exists also exists a Dynkin diagram automorphism in the group of Bäcklund transformations, however, we still do not know how these Dynkin diagram automorphisms manifest themselves in this theory. The associated linear problem, specified by Murata [17], is given by

$$
Y(q x)=A(x) Y(x),
$$

where

$$
A(x)=\left(\begin{array}{cc}
\left((x-y)(x-\alpha)+z_{1}\right) \kappa_{1} & \kappa_{2} w(x-y) \\
\frac{(x \gamma+\delta) \kappa_{1}}{w} & \left(x-y+z_{2}\right) \kappa_{2}
\end{array}\right) .
$$

We fix the determinant

$$
\operatorname{det} A(x)=\kappa_{1} \kappa_{2} x^{3},
$$

and let $A(0)$ possess one non-zero eigenvalue, $\lambda_{1}$. This allows us to specify the values

$$
\alpha=\frac{\lambda_{1}-z_{1} \kappa_{1}+\left(y-z_{2}\right) \kappa_{2}}{y \kappa_{1}}, \quad \gamma=\left(-2 y-\alpha+z_{2}\right) \kappa_{2}, \quad \delta=\frac{\left(y \alpha+z_{1}\right)\left(y-z_{2}\right) \kappa_{2}}{y} .
$$

We make a choice of parameterization of $z_{1}$ and $z_{2}$, given by

$$
z_{1}=\frac{y^{2}}{z}, \quad z_{2}=y z
$$

We will make a full correspondence between $y$ and $z$ with $f$ and $g$. To do this, let us first consider the full space of Schlesinger transformations on this space. 
The problem possesses formal solutions around $x=0$ and $x=\infty$ specified by

$$
\begin{aligned}
& Y_{0}(x)=\left(\left(\begin{array}{cc}
-1 & -w y \kappa_{2} \\
\frac{z-1}{w} & y(z-1) \kappa_{2}-\lambda
\end{array}\right)+O(x)\right)\left(\begin{array}{cc}
e_{q, \lambda_{1}}(x) & 0 \\
0 & \frac{e_{q, \lambda_{2}}(x)}{\theta_{q}(x)^{3}}
\end{array}\right), \\
& Y_{\infty}(x)=\left(I+\frac{1}{x}\left(\begin{array}{cc}
\frac{q(y+\alpha)}{q-1} & -\frac{w \kappa_{2}}{\kappa_{1}} \\
\frac{q \gamma}{w} & -\frac{q(y+\alpha)}{q-1}
\end{array}\right)+O\left(\frac{1}{x^{2}}\right)\right)\left(\begin{array}{cc}
\frac{e_{q, \kappa_{1}}(x)}{\theta_{q}(x)^{2}} & 0 \\
0 & \frac{e_{q, \kappa_{2}}(x)}{\theta_{q}(x)}
\end{array}\right) .
\end{aligned}
$$

The asymptotics of these solutions and the zeros of the determinant define the characteristic data to be

$$
M=\left\{\begin{array}{cc}
\kappa_{1} & \kappa_{2} \\
\lambda_{1} & \lambda_{2}
\end{array}\right\}
$$

meaning our co-ordinate system for the $A$ matrices should be

$$
M_{A}=\left\{\begin{array}{cc}
\kappa_{1} & \kappa_{2} \\
\lambda_{1} & \lambda_{2}
\end{array}: w, y, z\right\}
$$

where we have the constraint

$$
\lambda_{1} \lambda_{2}=\kappa_{1} \kappa_{2} .
$$

This specifies four parameters with one constraint, however, it is easy to verify on the level of parameters (and much more work to verify on the level of the Painlevé variables) that

$$
T_{\kappa_{1}, \lambda_{2}} \circ T_{\kappa_{1}, \lambda_{1}}^{-1} \circ T_{\kappa_{2}, \lambda_{1}}=T_{\kappa_{1}, \lambda_{2}},
$$

hence, we may regard $T_{\kappa_{1}, \lambda_{2}}$ as an element of the group generated by the other three elements. Upon further examination, the action of $R_{\kappa_{1}, \kappa_{2}, \lambda_{1}, \lambda_{2}}(x)=x I$ is represented by an identity on the Painlevé parameters, however, there is a $q$-shift of the asymptotic behaviors at 0 and $\infty$. We regard this as a trivial action, $T_{\kappa_{1}, \kappa_{2}, \lambda_{1}, \lambda_{2}}$, whereby, we have the relation

$$
T_{\kappa_{1}, \lambda_{2}} \circ T_{\kappa_{2}, \lambda_{1}}=T_{\kappa_{1}, \kappa_{2}, \lambda_{1}, \lambda_{2}},
$$

hence, if we include $T_{\kappa_{1}, \kappa_{2}, \lambda_{1}, \lambda_{2}}$, if we include $T_{\kappa_{1}, \kappa_{2}, \lambda_{1}, \lambda_{2}}$ in our generators, we need only consider the two non-trivial translations $T_{\kappa_{1}, \lambda_{1}}$ and $T_{\kappa_{1}, \lambda_{2}}$. We now proceed to calculate

$$
\begin{aligned}
& T_{\kappa_{1}, \lambda_{1}}:\left\{\begin{array}{ll}
\kappa_{1} & \kappa_{2} \\
\lambda_{1} & \lambda_{2}
\end{array}: w, y, z\right\} \rightarrow\left\{\begin{array}{cc}
q \kappa_{1} & \kappa_{2} \\
q \lambda_{1} & \lambda_{2}
\end{array}: \tilde{w}, \tilde{y}, \tilde{z}\right\}, \\
& T_{\kappa_{1}, \lambda_{2}}:\left\{\begin{array}{ll}
\kappa_{1} & \kappa_{2} \\
\lambda_{1} & \lambda_{2}
\end{array}: w, y, z\right\} \rightarrow\left\{\begin{array}{cc}
q \kappa_{1} & \kappa_{2} \\
\lambda_{1} & q \lambda_{2}
\end{array}: \tilde{w}, \tilde{y}, \tilde{z}\right\},
\end{aligned}
$$

where in these two cases, the action $(w, y, z) \rightarrow(\tilde{w}, \tilde{y}, \tilde{z})$, is obtained from the left action of a Schlesinger matrix, $R=R_{\kappa_{1}, \lambda_{1}}(x)$ and $R=R_{\kappa_{1}, \lambda_{2}}(x)$ respectively. One of the aspects of [20] was that $R(x)$ may be obtained directly from the solutions via expansions of

$$
R(x)=\tilde{Y}(x) Y(x)^{-1},
$$

where $Y=Y_{0}$ or $Y=Y_{\infty}$. Using $Y=Y_{0}$ an expansion of $R(x)$ around $x=0$ gives

$$
R(x)=R_{0}+O(x),
$$


where as using $Y=Y_{\infty}$ an expansion of $R$ around $x=\infty$ gives

$$
R(x)=\left(\begin{array}{ll}
x & 0 \\
0 & 0
\end{array}\right)+R_{0}+O\left(\frac{1}{x}\right) .
$$

The equality of these gives us that $R(x)$ is linear in $x$, in fact, expanding $R$ around $x=\infty$ to the constant term gives

$$
R(x)=\left(\begin{array}{cc}
x+\frac{q(\tilde{y}-y+\tilde{\alpha}-\alpha)}{q-1} & \frac{\kappa_{2} w}{\kappa_{1}} \\
\frac{q \tilde{\gamma}}{\tilde{w}} & 1
\end{array}\right) .
$$

This parameterization is the same for $R_{\kappa_{1}, \lambda_{1}}$ and $R_{\kappa_{1}, \lambda_{2}}$, however, $\tilde{y}$ and $\tilde{w}$ and hence $\tilde{\gamma}$ and $\tilde{\alpha}$ are different for each case. Computing the compatibility condition for the two non-trivial generators gives the following relations

$$
\begin{aligned}
& T_{\kappa_{1}, \lambda_{1}}: \tilde{w}=w\left(\frac{q \lambda_{1}(\tilde{z}-1)}{y \tilde{z} \kappa_{1}}\right), \quad \tilde{y}=\frac{q y \lambda \tilde{z}}{(\tilde{z}-1)\left(q \lambda+y \kappa_{2} \tilde{z}\right)}, \quad \tilde{z}=\frac{q z \lambda}{q y^{2} \kappa_{1}-y z \kappa_{2}}, \\
& T_{\kappa_{1}, \lambda_{2}}: \tilde{w}=w\left(\frac{\kappa_{2}}{\kappa_{1}}-\frac{q y}{z}\right), \quad \tilde{y}=\frac{\lambda_{1}}{q \kappa_{1} y-\kappa_{2} z}, \quad \tilde{z}=\frac{q \kappa_{1} y}{\kappa_{2} z} .
\end{aligned}
$$

We now note

$$
\mathbb{Z}^{3} \cong\left\langle T_{\kappa_{1}, \lambda_{1}}, T_{\kappa_{1}, \lambda_{2}}, T_{\kappa_{1}, \kappa_{2}, \lambda_{1}, \lambda_{2}}\right\rangle=G_{A},
$$

which is the lattice of connection preserving deformations.

We now specify the connection preserving deformation that defines $q-\mathrm{P}_{\mathrm{I}}$ as

$$
q-\mathrm{P}_{\mathrm{I}}:\left\{\begin{array}{cc}
\kappa_{1} & \kappa_{2} \\
\lambda_{1} & \lambda_{2}
\end{array}: w, y, z\right\} \rightarrow\left\{\begin{array}{cc}
\kappa_{1} & \kappa_{2} \\
q \lambda_{1} & \frac{\lambda_{2}}{q}
\end{array}: \tilde{w}, \tilde{y}, \tilde{z}\right\} .
$$

which is decomposed elementary Schlesinger transformations $q-\mathrm{P}_{\mathrm{I}}=T_{\kappa_{1}, \lambda_{1}} \circ T_{\kappa_{1}, \lambda_{2}}^{-1}$. A simple calculation reveals

$$
T_{\lambda_{1}, \lambda_{2}^{-1}}=T_{\kappa_{1}, \lambda_{1}} \circ T_{\kappa_{1}, \lambda_{2}}^{-1}: \tilde{w}=w(1-\tilde{z}), \quad \tilde{y} y=\frac{\lambda_{1} \tilde{z}}{\kappa_{1}(\tilde{z}-1)}, \quad \tilde{z} z=\frac{q \kappa_{1} y}{\kappa_{2}} .
$$

We make the correspondence with (4.1) by letting $f=\frac{\kappa_{2}}{q \kappa_{1} y}$ and $z=g, b_{1}=-\kappa_{2}^{2} /\left(q^{2} \lambda_{1} \kappa_{1}\right)$ and $b_{0} b_{1}=q$. In this way, the one and only translational element of $A_{1}^{(1)}$ is identified with $T_{\lambda_{1}, \lambda_{2}^{-1}}$.

\section{$4.2 \quad q-\mathrm{P}\left(A_{7}^{(1)^{\prime}}\right)$}

We note that there are many ways to interpret $q-\mathrm{P}\left(A_{7}^{(1)^{\prime}}\right)$. We choose but one element of infinite order that we are able to make a correspondence with, for this, in Sakai's notation, we choose $s_{1} \circ \sigma_{(1357)(2460)}$, which has the following effect

$$
\left\{\begin{array}{c}
b_{0} b_{1} \\
b_{2}
\end{array}: f, g\right\} \rightarrow\left\{\begin{array}{c}
\frac{b_{0}}{q} \frac{q}{b_{1}} \\
b_{2} b_{1}^{2}
\end{array}: \tilde{f}, \tilde{g}\right\}
$$

where

$$
\tilde{f} f=\frac{q+\tilde{g} b_{0}}{\tilde{g}(\tilde{g}+1) b_{0}}, \quad \tilde{g} g=\frac{q}{b_{0} b_{1} f^{2}},
$$


which is also known as $q-\mathrm{P}_{\mathrm{I}}^{\prime}$. The surface corresponds to the same affine Weyl group as before, however, the technical difference is that it corresponds to a copy of a root subsystem of $E_{8}^{(1)}$ where the lengths of the roots are different from those that correspond to $q-\mathrm{P}\left(A_{7}^{(1)}\right)$ [23].

The associated linear problem for $q-\mathrm{P}\left(A_{7}^{(1)^{\prime}}\right)$, has been given by Murata [17]. In terms of the theory presented above, the same theory applies in that the characteristic data is well defined, and the deformations are all prescribed in the same manner as for $q-\mathrm{P}\left(A_{7}^{(1)}\right)$. In short, we expect a three dimensional lattice of deformations as above. We take

$$
A(x)=\left(\begin{array}{cc}
\kappa_{1}\left((x-y)(x-\alpha)+z_{1}\right) & \kappa_{2} w(x-y) \\
\frac{\kappa_{1}}{w}(\gamma x+\delta) & \kappa_{2} z_{2}
\end{array}\right),
$$

where

$$
\alpha=\frac{-z_{1} \kappa_{1}-z_{2} \kappa_{2}+\lambda_{1}}{y \kappa_{1}}, \quad \gamma=z_{2} \kappa_{2}-\kappa_{2}, \quad \delta=y \gamma-y z_{2} \kappa_{2}-\alpha z_{2} \kappa_{2},
$$

and the choice of $z$ is specified by

$$
z_{1}=\frac{y^{2}}{z}, \quad z_{2}=z .
$$

We find the solutions are given by

$$
\begin{aligned}
Y_{0}(x)= & \left.\left(\begin{array}{cc}
-\frac{w y(1-z)}{z \kappa_{2}} & \frac{w y\left(y(z-1) \kappa_{2}-\lambda\right)}{\lambda_{1}-z \kappa_{2}} \\
1-z & y(z-1) \kappa_{2}-\lambda
\end{array}\right)+O(x)\right)\left(\begin{array}{cc}
e_{q, \lambda_{1}}(x) & 0 \\
0 & \frac{e_{q, \lambda_{2}}(x)}{\theta_{q}(x)^{2}}
\end{array}\right), \\
Y_{\infty}(x)= & \left(\begin{array}{cc}
I+\frac{1}{x}\left(\begin{array}{cc}
\frac{q(y+\alpha)}{q-1} & -\frac{w}{\kappa_{1}} \\
\frac{q \gamma}{w} & -\frac{q\left((z-1) \kappa_{1} y^{2}+z\left(\lambda_{1}-z \kappa_{2}\right)\right)}{(q-1) y z \kappa_{1}}
\end{array}\right)+O\left(\frac{1}{x^{2}}\right)
\end{array}\right) \\
& \times\left(\begin{array}{cc}
\frac{e_{q, \kappa_{1}}(x)}{\theta_{q}(x)^{2}} & 0 \\
0 & e_{q, \kappa_{2}}(x)
\end{array}\right)
\end{aligned}
$$

where

$$
\kappa_{1} \kappa_{2}=\lambda_{1} \lambda_{2} .
$$

This allows us to specify the characteristic data to be

$$
M=\left\{\begin{array}{cc}
\kappa_{1} & \kappa_{2} \\
\lambda_{1} & \lambda_{2}
\end{array}\right\}
$$

and the defining co-ordinate system for $A(x)$ to be

$$
M_{A}=\left\{\begin{array}{cc}
\kappa_{1} & \kappa_{2} \\
\lambda_{1} & \lambda_{2}
\end{array}: w, y, z\right\}
$$

For the same reasons as for the previous subsection, it suffices to define $T_{\kappa_{1}, \lambda_{1}}, T_{\kappa_{1}, \lambda_{2}}$ and $T_{\kappa_{1}, \kappa_{2}, \lambda_{1}, \lambda_{2}}$, which is a trivial shift induced by $R_{\kappa_{1}, \kappa_{2}, \lambda_{1}, \lambda_{2}}(x)=x I$. The matrices $R_{\kappa_{1}, \lambda_{1}}(x)$ and $R_{\kappa_{1}, \lambda_{2}}(x)$ are given by (4.2), allowing us compute the following:

$$
T_{\kappa_{1}, \lambda_{1}}: \tilde{w}=\frac{q w \lambda_{1}(\tilde{z}-1)}{y \kappa_{1} \tilde{z}}, \quad \tilde{y}=\frac{y \tilde{z}\left(q \lambda_{1}-\tilde{z} \kappa_{2}\right)}{q(\tilde{z}-1) \lambda_{1}}, \quad \tilde{z}=\frac{z \lambda_{1}}{y^{2} \kappa_{1}},
$$




$$
T_{\kappa_{1}, \lambda_{2}}: \tilde{w}=-q w \frac{y}{z}, \quad \tilde{y} y=\frac{\lambda_{1}}{q \kappa_{1}}, \quad \tilde{z} z=\frac{\lambda_{1}}{q \kappa_{2}} .
$$

In a similar manner to the associated linear problem for $\mathrm{P}\left(A_{7}^{(1)}\right)$, we have

$$
\mathbb{Z}^{3} \cong\left\langle T_{\kappa_{1}, \lambda_{1}}, T_{\kappa_{1}, \lambda_{2}}, T_{\kappa_{1}, \kappa_{2}, \lambda_{1}, \lambda_{2}}\right\rangle=G_{A}
$$

forming the lattice of connection preserving deformations.

We now identify the evolution of $q-\mathrm{P}_{\mathrm{I}}^{\prime}$ as a decomposition into the basis for elementary Schlesinger transformations above. $q-\mathrm{P}_{\mathrm{I}}^{\prime}=T_{\kappa_{1}, \lambda_{1}} \circ T_{\kappa_{1}, \lambda_{2}}^{-1}$. The action is specified by

$$
T_{\kappa_{1}, \lambda_{1}} \circ T_{\kappa_{1}, \lambda_{2}}^{-1}: \tilde{w}=w(1-\tilde{z}), \quad \tilde{y} y=\frac{\tilde{z}\left(q \lambda_{1}-\kappa_{2} \tilde{z}\right)}{q \kappa_{1}(\tilde{z}-1)}, \quad \tilde{z} z=\frac{q \kappa_{1}}{\kappa_{2}} y^{2},
$$

where we may identify with the above evolutions as $z=-1 / g, y=\sqrt{b_{0} b_{1}} f$ and $\kappa_{2} b_{0}=q^{2} \lambda_{1}-$ $q^{2} \kappa_{1}$.

\section{$4.3 \quad q-\mathrm{P}\left(A_{6}^{(1)}\right)$}

We now increase the dimension of the underlying lattice by introducing non-zero root of $\operatorname{det} A$. We note that in accordance with the notation of Sakai [23], we choose $\sigma \circ \sigma$ to define the evolution of $q-\mathrm{P}_{\mathrm{II}}$. This system may be equivalently written as

$$
\left\{\begin{array}{c}
b_{0} b_{1} \\
b_{2}
\end{array}: f, g\right\} \rightarrow\left\{\begin{array}{c}
b_{0} b_{1} \\
q b_{2}
\end{array}: \tilde{f}, \tilde{g}\right\}
$$

where $q=b_{0} b_{1}$ and

$$
\tilde{f} f=-\frac{b_{2} \tilde{g}}{\tilde{g}+b_{1} b_{2}}, \quad \tilde{g} g=b_{1} b_{2}(1-f) .
$$

We find a parameterization of the associated linear problem to be given by

$$
A(x)=\left(\begin{array}{cc}
\kappa_{1}\left((x-\alpha)(x-y)+z_{1}\right) & \kappa_{2} w(x-y) \\
\frac{\kappa_{1}}{w}(\gamma x+\delta) & \kappa_{2}\left(x-y+z_{2}\right)
\end{array}\right),
$$

where by letting

$$
\operatorname{det} A(x)=\kappa_{1} \kappa_{2} x^{2}\left(x-a_{1}\right),
$$

we readily find the entries of $A(x)$ are given by

$$
\alpha=\frac{-z_{1} \kappa_{1}+\left(y-z_{2}\right) \kappa_{2}+\lambda_{1}}{y \kappa_{1}}, \quad \gamma=-2 y-\alpha+a_{1}+z_{2}, \quad \delta=\frac{\left(y \alpha+z_{1}\right)\left(y-z_{2}\right)}{y} .
$$

We take a choice of $z$ to be specified by

$$
z_{1}=\frac{y\left(y-a_{1}\right)}{z}, \quad z_{2}=y z .
$$

The formal symbolic solutions are specified by

$$
Y_{0}(x)=\left(\left(\begin{array}{cc}
-\frac{w(1-z)}{z-1} & -w y \kappa_{2} \\
1-z & y(z-1) \kappa_{2}-\lambda_{1}
\end{array}\right)+O(x)\right)\left(\begin{array}{cc}
e_{q, \lambda_{1}}(x) & 0 \\
0 & \frac{e_{q, \lambda_{2}}(x)}{\theta_{q}(x)^{2}}
\end{array}\right)
$$




$$
\begin{aligned}
Y_{\infty}(x)= & \left(I+\frac{1}{x}\left(\begin{array}{cc}
\frac{q(y+\alpha)}{q-1} & -\frac{w \kappa_{2}}{\kappa_{1}} \\
\frac{q \gamma}{w} & -\frac{q y(z-1)\left(\left(y-a_{1}\right) \kappa_{1}-z \kappa_{2}\right)+q z \lambda_{1}}{(q-1) y z \kappa_{1}}
\end{array}\right)+O\left(\frac{1}{x^{2}}\right)\right) \\
& \times\left(\begin{array}{cc}
\frac{e_{q, \kappa_{1}}(x)}{\theta_{q}(x)^{2}} & 0 \\
0 & \frac{e_{q, \kappa_{2}}(x)}{\theta_{q}(x)}
\end{array}\right)
\end{aligned}
$$

and the zeros of $\operatorname{det} A(x)$ define the characteristic data to be

$$
M=\left\{\begin{array}{ccc}
\kappa_{1} & \kappa_{2} & \\
\lambda_{1} & \lambda_{2} & a_{1}
\end{array}\right\}
$$

This allows us to write co-ordinate system for $A$ as

$$
M_{A}=\left\{\begin{array}{ccc}
\kappa_{1} & \kappa_{2} & \\
\lambda_{1} & \lambda_{2} & a_{1}
\end{array}: w, y, z\right\}
$$

where we have the constraint

$$
\lambda_{1} \lambda_{2}=-a_{1} \kappa_{1} \kappa_{2} .
$$

For the same reasons as previous subsections, it suffices to define $T_{\kappa_{1}, \lambda_{1}}, T_{\kappa_{1}, \lambda_{1}}$ and $T_{\kappa_{1}, \kappa_{2}, \lambda_{1}, \lambda_{2}}$, however, we also need to choose an element that changes $a_{1}$. We choose $T_{a_{1}, \lambda_{1}}$, which is induced by some $R_{a_{1}, \lambda_{1}}$. By solving the scalar determinantal problem, one arrives at the conclusion

$$
\operatorname{det} R_{a_{1}, \lambda_{1}}=\frac{x}{x-q a_{1}} .
$$

When we consider the change in $\kappa_{i}$ values, it is clear that by expanding $R_{a_{1}, \lambda_{1}}$ around $x=\infty$ using $R_{a_{1}, \lambda_{1}}=\tilde{Y}_{\infty} Y_{\infty}^{-1}$ we have the expansion

$$
R_{a_{1}, \lambda_{1}}=I+\frac{1}{x}\left(\begin{array}{cc}
\frac{q(y+\alpha)}{q-1} & -\frac{w \kappa_{2}}{\kappa_{1}} \\
\frac{q \gamma}{w} & -\frac{q\left(y+\alpha-a_{1}\right)}{q-1}
\end{array}\right)+O\left(\frac{1}{x^{2}}\right) .
$$

This means that we may deduce that $R_{a_{1}, \lambda_{1}}$ is given by

$$
R_{a_{1}, \lambda_{1}}=\frac{1}{x-q a_{1}}\left(\begin{array}{cc}
x-\frac{q(y-\tilde{y}+\alpha-\tilde{\alpha})}{q-1}-q a_{1} & \frac{\kappa_{2}(w-\tilde{w})}{\kappa_{1}} \\
\frac{q(\tilde{w} \gamma-w \tilde{\gamma})}{w \tilde{w}} & x+\frac{q(y-\tilde{y}+\alpha-\tilde{\alpha})}{q-1}
\end{array}\right) .
$$

We can take $R_{\kappa_{1}, \lambda_{1}}$ and $R_{\kappa_{1}, \lambda_{2}}$ to be of the form of (4.2). Computing the compatibility reveals

$$
\begin{aligned}
& T_{a_{1}, \lambda_{1}}: \tilde{w}=w(1-\tilde{z}), \quad \tilde{y} y=\frac{\tilde{z} \lambda_{1}}{(\tilde{z}-1) \kappa_{1}}, \quad \tilde{z} z=\frac{q\left(y-a_{1}\right) \kappa_{1}}{\kappa_{2}}, \\
& T_{\kappa_{1}, \lambda_{1}}: \tilde{w}=\frac{w\left(q \lambda_{1}-\kappa_{2} \tilde{y}(\tilde{z}-1)\right)}{\kappa_{1} \tilde{y}}, \quad \tilde{y} y=\frac{z \lambda_{1}}{\kappa_{1}(\tilde{z}-1)}, \quad \tilde{z}=\frac{q y a_{1} \kappa_{1}+q z \lambda_{1}}{q y^{2} \kappa_{1}-y z \kappa_{2}}, \\
& T_{\kappa_{1}, \lambda_{2}}: \quad \tilde{w}=\frac{w \kappa_{2}(1-\tilde{z})}{\kappa_{1}}, \quad \tilde{y} y=\frac{\lambda_{1} \tilde{z}}{q \kappa_{1}(\tilde{z}-1)}, \quad \tilde{z} z=\frac{q\left(y-a_{1}\right) \kappa_{1}}{\kappa_{2}} .
\end{aligned}
$$


The full group of Schlesinger transformations is given by

$$
\mathbb{Z}^{4} \cong\left\langle T_{a_{1}, \lambda_{1}}, T_{\kappa_{1}, \lambda_{1}}, T_{\kappa_{1}, \lambda_{2}}, T_{\kappa_{1}, \kappa_{2}, \lambda_{1}, \lambda_{2}}\right\rangle=G_{A},
$$

which is the lattice of connection preserving deformations.

To obtain a full correspondence of $T_{a_{1}, \lambda_{1}}$ with (4.3) we let

$$
y=a_{1} f, \quad z=-g \frac{b_{0}}{b_{2}},
$$

with the relations $b_{0}=\frac{\lambda_{1}}{a_{1} \kappa_{2}}$ and $b_{2}=-\frac{\lambda_{1}}{q a_{1}^{2} \kappa_{1}}$. We know that the action of $T_{a_{1}, \lambda_{1}} \circ T_{\kappa_{1}, \lambda_{2}^{-1}}$ is trivial. I.e., we have

$$
T_{a_{1}, \lambda_{1}} \circ T_{\kappa_{1}, \lambda_{2}^{-1}}: \tilde{w}=\frac{\kappa_{1} w}{\kappa_{2}}, \quad \tilde{y}=q y, \quad \tilde{z}=z,
$$

which leaves $f$ and $g$ invariant. When we restrict our attention to $f$ and $g$ alone we only have a two dimensional lattice of transformations, corresponding to $\left(A_{1}+A_{1}\right)^{(1)}$.

\section{$4.4 \quad q-\mathrm{P}\left(A_{5}^{(1)}\right)$}

As a natural progression from the above cases, we allow two non-zero roots of the determinant. In doing so, we obtain an associated linear problem for both $q-\mathrm{P}_{\mathrm{III}}$ and $q-\mathrm{P}_{\mathrm{IV}}$. We also introduce a symmetry as one is able to naturally permute the two roots of the determinant, which we will formalize later. We turn to the particular presentation of Noumi et al. [18]. We present a version of $q-\mathrm{P}_{\mathrm{III}}$ as

$$
\left\{\begin{array}{c}
b_{0} b_{1} \\
b_{2} c
\end{array}: f, g\right\} \rightarrow\left\{\begin{array}{c}
q b_{0} b_{1} / q \\
b_{2} c
\end{array}: \tilde{f}, \tilde{g}\right\},
$$

where $q=b_{0} b_{1} b_{2}$ and

$$
\tilde{f}=\frac{q c}{f g} \frac{1+g b_{0}}{g+b_{0}}, \quad \tilde{g}=\frac{q c^{2}}{g \tilde{f}} \frac{b_{1}+q \tilde{f}}{q+b_{1} \tilde{f}} .
$$

Using the same variables, a representation of $q-\mathrm{P}_{\mathrm{IV}}$ is given by

$$
\left\{\begin{array}{r}
b_{0} b_{1} \\
b_{2} c
\end{array}: f, g\right\} \rightarrow\left\{\begin{array}{l}
b_{0} b_{1} \\
b_{2} q c
\end{array}: \tilde{f}, \tilde{g}\right\}
$$

where

$$
\tilde{f} f=\frac{c^{2} q b_{1}\left(1+g b_{0}+f g b_{1} b_{0}\right) b_{2}}{q b_{1} b_{2} c^{2}+g+f g b_{1}}, \quad \tilde{g} g=\frac{b_{0} b_{1}\left(q\left(g b_{0}+1\right) b_{2} c^{2}+f g\right)}{\left(g b_{0}\left(f b_{1}+1\right)+1\right)} .
$$

These two equations are interesting as a pair as they have the same surface of initial conditions. If any natural correspondence was to be sought between the theory of the associated linear problems and the theory of the surfaces of initial conditions [23], these two equations should possess the same associated linear problem up to reparameterizations. It is interesting to note that the associated linear problems defined by [17] do indeed possess the same characteristic data and asymptotics. We unify them by writing them as one linear problem, given by

$$
A(x)=\left(\begin{array}{cc}
\kappa_{2}\left((x-\alpha)(x-y)+z_{1}\right) & \kappa_{2} w(x-y) \\
\frac{\kappa_{1}}{w}(\gamma x+\delta) & \kappa_{2}\left(x-y+z_{2}\right)
\end{array}\right),
$$


where

$$
\operatorname{det} A(x)=\kappa_{1} \kappa_{2} x\left(x-a_{1}\right)\left(x-a_{2}\right) .
$$

This determinant and allowing $A(0)$ to have one non-zero eigenvalue, $\lambda_{1}$, allows us to specify the parameterization as

$$
\alpha=\frac{-z_{1} \kappa_{1}+\left(y-z_{2}\right) \kappa_{2}+\lambda_{1}}{y \kappa_{1}}, \quad \gamma=a_{1}+a_{2}+z_{2}-2 y-\alpha, \quad \delta=\frac{\left(y \alpha+z_{1}\right)\left(y-z_{2}\right)}{y} .
$$

We choose $z$ to be specified by

$$
z_{1}=\frac{y\left(y-a_{1}\right)}{z}, \quad z_{2}=z\left(y-a_{2}\right) .
$$

The formal solutions are given by

$$
\begin{aligned}
& Y_{0}(x)=\left(\left(\begin{array}{cc}
w y & w y \kappa_{2} \\
y-z y+z a_{2} & \left(-z y+y+z a_{2}\right) \kappa_{2}+\lambda_{1}
\end{array}\right)+O(x)\right)\left(\begin{array}{cc}
e_{q, \lambda_{1}}(x) & 0 \\
0 & \frac{e_{q, \lambda_{2}}(x)}{\theta_{q}(x)}
\end{array}\right), \\
& Y_{\infty}(x)=\left(I+\frac{1}{x}\left(\begin{array}{cc}
\frac{q(y+\alpha)}{q-1} & -\frac{w \kappa_{2}}{\kappa_{1}} \\
\frac{q \gamma}{w} & -\frac{q\left(y+\alpha-a_{1}-a_{2}\right)}{q-1}
\end{array}\right)+O\left(\frac{1}{x^{2}}\right)\right)\left(\begin{array}{cc}
\frac{e_{q, \kappa_{1}}(x)}{\theta_{q}(x)^{2}} & 0 \\
0 & \frac{e_{q, \kappa_{2}}(x)}{\theta_{q}(x)}
\end{array}\right),
\end{aligned}
$$

which is enough to define

$$
M=\left\{\begin{array}{llll}
\kappa_{1} & \kappa_{2} & \lambda_{1} & \lambda_{2} \\
a_{1} & a_{2} & &
\end{array}\right\}
$$

hence, the co-ordinates for $A(x)$ may be stated as

$$
M_{A}=\left\{\begin{array}{lllll}
\kappa_{1} & \kappa_{2} & \lambda_{1} & \lambda_{2} & : w, y, z \\
a_{1} & a_{2} & &
\end{array}\right\}
$$

We notice that there is a natural symmetry introduced in the parameterization, that is that $A$ is left invariant under the action

$$
s_{1}:\left\{\begin{array}{cccc}
\kappa_{1} & \kappa_{2} & & \\
\lambda_{1} & \lambda_{2} & a_{1} & a_{2}
\end{array}: w, y, z\right\} \rightarrow\left\{\begin{array}{cccc}
\kappa_{1} & \kappa_{2} & & \\
\lambda_{1} & \lambda_{2} & a_{2} & a_{1}
\end{array}: w, y, z \frac{y-a_{2}}{y-a_{1}}\right\} .
$$

If we include $s_{1}$ as a symmetry, we need only specify $T_{a_{1}, \lambda_{1}}, T_{\kappa_{1}, \lambda_{1}}$ and $T_{\kappa_{1}, \lambda_{2}}$ as we may exploit this symmetry to obtain $T_{a_{2}, \lambda_{1}}=s_{1} \circ T_{a_{1}, \lambda_{1}} \circ s_{1}$. The matrix $R_{a_{1}, \lambda_{1}}$ is of the form (4.4) and the matrices $R_{\kappa_{1}, \lambda_{1}}$ and $R_{\kappa_{1}, \lambda_{2}}$ are of the form (4.2). We may use the compatibility conditions to obtain the following correspondences:

$$
\begin{aligned}
& T_{a_{1}, \lambda_{1}}: \tilde{w}=w(1-\tilde{z}), \quad \tilde{y} y=\frac{\tilde{z}\left(\tilde{z} a_{2} \kappa_{2}+q \lambda_{1}\right)}{q(\tilde{z}-1) \kappa_{1}}, \quad \tilde{z} z=\frac{q y\left(y-a_{1}\right) \kappa_{1}}{\left(y-a_{2}\right) \kappa_{2}}, \\
& T_{\kappa_{1}, \lambda_{1}}: \tilde{w}=\frac{w\left(q y \kappa_{1}-z \kappa_{2}\right)(\tilde{z}-1)}{z \kappa_{1}}, \quad \tilde{y} y=\frac{z\left(q \lambda_{1}+a_{2} \kappa_{2} \tilde{z}\right)}{q \kappa_{1}(\tilde{z}-1)}, \quad \tilde{z}=\frac{q y a_{1} \kappa_{1}+q z \lambda_{1}}{q y^{2} \kappa_{1}-y z \kappa_{2}}, \\
& T_{\kappa_{1}, \lambda_{2}}: \tilde{w}=w\left(\frac{q\left(a_{1}-y\right)}{z}+\frac{\kappa_{2}}{\kappa_{1}}\right), \quad \tilde{y} y=\frac{\left(z a_{2} \kappa_{2}+\lambda_{1}\right) \tilde{z}}{q \kappa_{1}(\tilde{z}-1)}, \\
& \tilde{z} z=-\frac{q \kappa_{1}\left(z a_{1} a_{2} \kappa_{2}+\left(a_{1}-y\right) \lambda_{1}\right)}{\kappa_{2}\left(a_{2}\left(z \kappa_{2}-q y \kappa_{1}\right)+\lambda_{1}\right)} .
\end{aligned}
$$


We may now specify $T_{a_{2}, \lambda_{1}}=s_{1} \circ T_{a_{1}, \lambda_{1}} \circ s_{1}$.

$$
T_{a_{2}, \lambda_{1}}: \tilde{w}=w\left(1-\frac{q y \kappa_{1}}{z \kappa_{2}}\right), \quad \tilde{y}=\frac{q y a_{1} \kappa_{1}+q z \lambda_{1}}{q y z \kappa_{1}-z^{2} \kappa_{2}}, \quad \tilde{z} z=\frac{q y \kappa_{1}\left(\tilde{y}-a_{1}\right)}{\kappa_{2}\left(\tilde{y}-q a_{2}\right)} .
$$

This gives us the full lattice

$$
\mathbb{Z}^{5} \cong\left\langle T_{a_{1}, \lambda_{1}}, T_{a_{2}, \lambda_{1}}, T_{\kappa_{1}, \lambda_{1}}, T_{\kappa_{1}, \lambda_{2}}, T_{\kappa_{1}, \kappa_{2}, \lambda_{1}, \lambda_{2}}\right\rangle=G_{A} .
$$

We identify the evolution of $q-\mathrm{P}_{\mathrm{III}}$ with $T_{a_{1}, \lambda_{1}}$ under the change of variables

$$
y=\frac{q b_{0} b_{1}^{2} \lambda_{1}}{g \kappa_{2}}, \quad z=-\frac{f}{q b_{1}}
$$

so long as the following relations hold:

$$
b_{0}^{2}=\frac{a_{1}}{a_{2}}, \quad \lambda_{2}=c^{2} \kappa_{2}, \quad \kappa_{2} a_{2}=-q b_{1}^{2} \lambda_{1} .
$$

The symmetry between $a_{1}$ and $a_{2}$ alternates between the two translational components of the lattice of translations.

With the above identification, we also deduce $q-\mathrm{P}_{\mathrm{VI}}$ may be identified with

$$
\begin{gathered}
T_{a_{1}, \lambda_{1}} \circ T_{a_{2}, \lambda_{1}} \circ T_{\kappa_{1}, \lambda_{2}} \circ T_{\kappa_{1}, \lambda_{1}}^{-1}: \tilde{w}=w-\frac{q w\left(y-a_{1}\right) \kappa_{1}}{z \kappa_{2}}, \quad \tilde{y}=\frac{q\left(y-a_{1}\right) \lambda_{1}-q z a_{1} a_{2} \kappa_{2}}{y\left(q\left(y-a_{1}\right) \kappa_{1}-z \kappa_{2}\right)}, \\
\tilde{z}=-\frac{q \kappa_{1}\left(z a_{1} a_{2} \kappa_{2}+\left(a_{1}-y\right) \lambda_{1}\right)}{z \kappa_{2}\left(a_{2}\left(z \kappa_{2}-q y \kappa_{1}\right)+\lambda_{1}\right)} .
\end{gathered}
$$

We also note that in addition to $T_{\kappa_{1}, \kappa_{2}, \lambda_{1}, \lambda_{2}}$ being trivial, we have that according to the variables $f$ and $g$, the transformation $T_{\kappa_{1}, \lambda_{1}^{-1}, a_{1}^{-1}, a_{2}^{-1}}=T_{a_{1}, \lambda_{1}}^{-1} \circ T_{\kappa_{1}, \lambda_{1}} \circ T_{a_{2}, \lambda_{1}}^{-1}$ is also trivial.

\section{$4.5 \quad q-\mathrm{P}\left(A_{4}^{(1)}\right)$}

The next logical step in the progression of associated linear problems is to assume that the determinant has three non-zero roots. This brings us to the case of $q-\mathrm{P}_{\mathrm{V}}$ :

$$
\left\{\begin{array}{ccc}
b_{0} b_{1} & b_{2} \\
b_{3} & b_{4}
\end{array}: f, g\right\} \rightarrow\left\{\begin{array}{ccc}
q b_{0} b_{1} b_{2} \\
b_{3} b_{4} / q
\end{array}: \tilde{f}, \tilde{g}\right\}
$$

where

$$
f \tilde{f}=\frac{b_{0}+g b_{2}}{b_{0}\left(g b_{2}+1\right)\left(g b_{1} b_{2}+1\right) b_{3}}, \quad g \tilde{g}=\frac{(\tilde{f}-1) b_{0}\left(\tilde{f} b_{3}-1\right)}{\tilde{f} b_{2}\left(\tilde{f} b_{0} b_{1} b_{2} b_{3}-1\right)} .
$$

The associated linear problem was introduced by Murata [17], and the particular group has been explored in connection with the big $q$-Laguerre polynomials [20]. This case introduces two new symmetries; the first is that we have the symmetric group on the three $\operatorname{roots}$ of $\operatorname{det} A$, and the other is that the two solutions at $x=0$ are of the same order. The result is that they are interchangeable on the level of solutions of a single second order $q$-difference equation. The offshoot of this is, by including extra symmetries, we need only compute two non-trivial connection preserving deformations.

Let us proceed by first giving a parameterization of the associated linear problem. We let

$$
A(x)=\left(\begin{array}{cc}
\kappa_{1}\left((x-\alpha)(x-y)+z_{1}\right) & \kappa_{2} w(x-y) \\
\frac{\kappa_{1}}{w}(\gamma x+\delta) & \kappa_{2}\left(x-y+z_{2}\right)
\end{array}\right),
$$


where

$$
\operatorname{det} A(x)=\kappa_{1} \kappa_{2}\left(x-a_{1}\right)\left(x-a_{2}\right)\left(x-a_{3}\right) .
$$

Differently to previous case, the determinant of $A(0)$ is non-zero, hence, we require that the eigenvalues of $A(0), \lambda_{1}$ and $\lambda_{2}$, are both not necessarily zero. This determines that the parameters in $A(x)$ are specified by

$$
\begin{aligned}
& \alpha=\frac{-z_{1} \kappa_{1}+y \kappa_{2}-z_{2} \kappa_{2}+\lambda_{1}+\lambda_{2}}{y \kappa_{1}}, \quad \gamma=-2 y-\alpha+a_{1}+a_{2}+a_{3}+z_{2}, \\
& \delta=\frac{\left(y \alpha+z_{1}\right)\left(y-z_{2}\right)-a_{1} a_{2} a_{3}}{y}
\end{aligned}
$$

where we choose our parameter $z$ to be specified by

$$
z_{1}=\frac{\left(y-a_{1}\right)\left(y-a_{2}\right)}{z}, \quad z_{2}=z\left(y-a_{3}\right) .
$$

Our constraint is now

$$
\kappa_{1} \kappa_{2} a_{1} a_{2} a_{3}=-\lambda_{1} \lambda_{2}
$$

The fundamental solutions are of the form

$$
\begin{aligned}
Y_{0}(x)= & \left(\left(\begin{array}{cc}
-w y & -w y \kappa_{2} \\
y(z-1)-z a_{3}-\frac{\lambda_{2}}{\kappa_{2}} & \left(y(z-1)-z a_{3}\right) \kappa_{2}-\lambda_{1}
\end{array}\right)+O(x)\right) \\
& \times\left(\begin{array}{cc}
e_{q, \lambda_{1}}(x) & 0 \\
0 & e_{q, \lambda_{2}}(x)
\end{array}\right), \\
Y_{\infty}(x)= & \left(\begin{array}{cc}
I+\frac{1}{x}\left(\begin{array}{cc}
\frac{q(y+\alpha)}{q-1} & -\frac{w \kappa_{2}}{\kappa_{1}} \\
\frac{q \gamma}{w} & -\frac{q\left(y+\alpha-a_{1}-a_{2}-a_{3}\right)}{q-1}
\end{array}\right)+O\left(\frac{1}{x^{2}}\right)
\end{array}\right) \\
& \times\left(\begin{array}{cc}
\frac{e_{q, \kappa_{1}}(x)}{\theta_{q}(x)^{2}} & 0 \\
0 & \frac{e_{q, \kappa_{2}}(x)}{\theta_{q}(x)}
\end{array}\right) .
\end{aligned}
$$

This specifies that our characteristic data may be taken to be

$$
M=\left\{\begin{array}{llll}
a_{1} & a_{2} & a_{3} & \\
\kappa_{1} & \kappa_{2} & \lambda_{1} & \lambda_{2}
\end{array}\right\}
$$

and hence, our co-ordinate space for $A(x)$ is given by

$$
M_{A}=\left\{\begin{array}{llll}
a_{1} & a_{2} & a_{3} \\
\kappa_{1} & \kappa_{2} & \lambda_{1} & \lambda_{2}
\end{array}: w, y, z\right\}
$$

We identify immediately that the parametrization is invariant under the following transformations

$$
s_{0}:\left\{\begin{array}{cccc}
a_{1} & a_{2} & a_{3} \\
\kappa_{1} & \kappa_{2} & \lambda_{1} & \lambda_{2}
\end{array}: w, y, z\right\} \rightarrow\left\{\begin{array}{cccc}
a_{1} & a_{2} & a_{3} & \\
\kappa_{1} & \kappa_{2} & \lambda_{2} & \lambda_{1}
\end{array}: w, y, z\right\}
$$




$$
\begin{aligned}
& s_{1}:\left\{\begin{array}{cccc}
a_{1} & a_{2} & a_{3} \\
\kappa_{1} & \kappa_{2} & \lambda_{1} & \lambda_{2}
\end{array}: w, y, z\right\} \rightarrow\left\{\begin{array}{llll}
a_{2} & a_{1} & a_{3} & \\
\kappa_{1} & \kappa_{2} & \lambda_{1} & \lambda_{2}
\end{array}: w, y, z\right\}, \\
& s_{2}:\left\{\begin{array}{llll}
a_{1} & a_{2} & a_{3} \\
\kappa_{1} & \kappa_{2} & \lambda_{1} & \lambda_{2}
\end{array}: w, y, z\right\} \rightarrow\left\{\begin{array}{llll}
a_{1} & a_{3} & a_{2} & \\
\kappa_{1} & \kappa_{2} & \lambda_{1} & \lambda_{2}
\end{array}: w, y, z \frac{y-a_{3}}{y-a_{2}}\right\} .
\end{aligned}
$$

If we now specify $T_{\kappa_{1}, \lambda_{1}}$, the trivial transformation, $T_{\kappa_{1}, \kappa_{2}, \lambda_{1}, \lambda_{2}}$ and $T_{a_{1}, \lambda_{1}}$, we may obtain a generating set using the symmetries to obtain

$$
T_{a_{2}, \lambda_{1}}=s_{1} \circ T_{a_{1}, \lambda_{1}} \circ s_{1}, \quad T_{a_{3}, \lambda_{1}}=s_{2} \circ s_{1} \circ T_{a_{1}, \lambda_{1}} \circ s_{1} \circ s_{2}, \quad T_{\kappa_{1}, \lambda_{2}}=s_{0} \circ T_{\kappa_{1}, \lambda_{1}} \circ s_{0} .
$$

The transformations $T_{\kappa_{1}, \lambda_{1}}$ and $T_{a_{1}, \lambda_{1}}$ are specified by the relations

$$
\begin{aligned}
T_{a_{1}, \lambda_{1}}: \tilde{w} & =\frac{w\left(\left(y-a_{1}\right) a_{2} a_{3} \kappa_{1} \kappa_{2}+\left(q y\left(y-a_{1}\right) \kappa_{1}+z\left(a_{3}-y\right) \kappa_{2}\right) \lambda_{1}\right)}{\kappa_{2}\left(\left(y-a_{1}\right) a_{2} a_{3} \kappa_{1}+z\left(a_{3}-y\right) \lambda_{1}\right)}, \\
\tilde{y} & =-\frac{a_{2}(w-\tilde{w})\left(q \lambda_{1}+a_{3} \kappa_{2} \tilde{z}\right)}{q \lambda_{1}(\tilde{w}+w(\tilde{z}-1))} \\
\tilde{z} & =\frac{z \lambda_{1}(w-\tilde{w})+a_{2} \kappa_{1}\left(w\left(y-a_{1}\right)+\left(y(z-1)+a_{1}\right) \tilde{w}\right)}{w\left(y-a_{1}\right) a_{2} \kappa_{1}+w z \lambda_{1}} \\
T_{\kappa_{1}, \lambda_{1}}: \tilde{w} & =\frac{w\left(-q \kappa_{1} y^{2}+\left(q\left(a_{1}+a_{2}\right) \kappa_{1}+z \kappa_{2}\right) y-q a_{1} a_{2} \kappa_{1}+q z \lambda_{1}\right)}{y z \kappa_{1}}, \\
\tilde{y} & =\frac{q \lambda_{1}+a_{3} \kappa_{2} \tilde{z}}{q \psi \kappa_{1} \lambda_{1}-q \psi \kappa_{1} \lambda_{1} \tilde{z}}, \quad \tilde{z}=\frac{q\left(\psi a_{1} \kappa_{1}-1\right)\left(\psi a_{2} \kappa_{1}-1\right)}{z \psi\left(\kappa_{2}+q \psi \kappa_{1} \lambda_{1}\right)}
\end{aligned}
$$

where we have introduced

$$
\psi=\frac{y}{a_{1} a_{2} \kappa_{1}-z \lambda_{1}}
$$

for convenience. We may now identify the lattice of connection preserving deformations as

$$
\mathbb{Z}^{6} \cong\left\langle T_{\kappa_{1}, \lambda_{1}}, T_{\kappa_{1}, \lambda_{2}}, T_{\kappa_{1}, \kappa_{2}, \lambda_{1}, \lambda_{2}}, T_{a_{1}, \lambda_{1}}, T_{a_{2}, \lambda_{1}}, T_{a_{3}, \lambda_{1}}\right\rangle=G_{A}
$$

We may identify the evolution of $q-\mathrm{P}_{\mathrm{V}}$ with the Schlesinger transformation

$$
\begin{aligned}
q-\mathrm{P}_{\mathrm{V}}=T_{a_{1}, a_{2}, \lambda_{1}, \lambda_{2}}: & \tilde{w}=w(1-\tilde{z}), \quad \tilde{y} y=\frac{\left(q \lambda_{1}+a_{3} \kappa_{2} \tilde{z}\right)\left(\lambda_{1} \tilde{z}-q a_{1} a_{2} \kappa_{1}\right)}{q \kappa_{1} \lambda_{1}(\tilde{z}-1)} \\
\tilde{z} z & =\frac{q\left(y-a_{1}\right)\left(y-a_{2}\right) \kappa_{1}}{\left(y-a_{3}\right) \kappa_{2}}
\end{aligned}
$$

where the correspondence between $y$ and $z$ with $f$ and $g$ is given by

$$
y=\frac{a_{2}}{f}, \quad z=-\frac{b_{2}}{b_{0}} g
$$

under the relations

$$
a_{1}=a_{2} b_{3}, \quad a_{2}=a_{3} b_{4}, \quad q a_{2} b_{0} \kappa_{1}=-b_{2} \kappa_{2}, \quad b_{0} b_{1} b_{4} \lambda_{1}=a_{2} \kappa_{2} .
$$

In addition to $T_{\kappa_{1}, \kappa_{2}, \lambda_{1}, \lambda_{2}}$ being trivial, we also have that the transformation $T_{a_{1}, a_{2}, a_{3}, \kappa_{1}^{-1}, \lambda_{1}, \lambda_{2}}$ is trivial. 


\section{$4.6 \quad q-\mathrm{P}\left(A_{3}^{(1)}\right)$}

This particular case was originally the subject of the article by Jimbo and Sakai [14], which introduced the idea of the preservation of a connection matrix [3]. The article was also responsible for introducing a discrete analogue of the sixth Painlevé equation [14], given by

$$
\left\{\begin{array}{llll}
b_{1} & b_{2} & b_{3} & b_{4} \\
b_{5} & b_{6} & b_{7} & b_{8}
\end{array}: f, g\right\} \rightarrow\left\{\begin{array}{llll}
q b_{1} & q b_{2} & b_{3} & b_{4} \\
q b_{5} & q b_{6} & b_{7} & b_{8}
\end{array}: \tilde{f}, \tilde{g}\right\}
$$

where

$$
\frac{\tilde{f} f}{b_{7} b_{8}}=\frac{\tilde{g}-q b_{1}}{\tilde{g}-b_{3}} \frac{\tilde{g}-q b_{2}}{\tilde{g}-b_{4}}, \quad \frac{\tilde{g} g}{b_{3} b_{4}}=\frac{f-b_{5}}{f-b_{7}} \frac{f-b_{6}}{f-b_{8}},
$$

where

$$
q=\frac{b_{3} b_{4} b_{5} b_{6}}{b_{1} b_{2} b_{7} b_{8}}
$$

The way in which $q-\mathrm{P}_{\mathrm{VI}}$, or $q-\mathrm{P}\left(A_{3}^{(1)}\right)$, was derived by Jimbo and Sakai [14] mirrors the theory that led to the formulation of $\mathrm{P}_{\mathrm{VI}}$ by Fuchs via the classical theory of monodromy preserving deformations [9, 8]. The associated linear problem originally derived by Jimbo and Sakai [14] is given by

$$
A(x)=\left(\begin{array}{cc}
\kappa_{1}\left((x-y)(x-\alpha)+z_{1}\right) & \kappa_{2} w(x-y) \\
\frac{\kappa_{1}}{w}(\gamma x+\delta) & \kappa_{2}\left((x-y)(x-\beta)+z_{2}\right)
\end{array}\right),
$$

where letting the eigenvalues of $A(0)$ be $\lambda_{1}$ and $\lambda_{2}$, and the determinant of $A(x)$ be

$$
\operatorname{det} A(x)=\kappa_{1} \kappa_{2}\left(x-a_{1}\right)\left(x-a_{2}\right)\left(x-a_{3}\right)\left(x-a_{4}\right),
$$

leads to

$$
\begin{aligned}
& \alpha=\frac{-z_{1} \kappa_{1}-\left(y\left(-2 y+a_{1}+a_{2}+a_{3}+a_{4}\right)+z_{2}\right) \kappa_{2}+\lambda_{1}+\lambda_{2}}{y\left(\kappa_{1}-\kappa_{2}\right)}, \\
& \beta=\frac{\left(y\left(-2 y+a_{1}+a_{2}+a_{3}+a_{4}\right)+z_{1}\right) \kappa_{1}+z_{2} \kappa_{2}-\lambda_{1}-\lambda_{2}}{y\left(\kappa_{1}-\kappa_{2}\right)}, \\
& \gamma=y^{2}+2(\alpha+\beta) y+\alpha \beta-a_{2} a_{3}-\left(a_{2}+a_{3}\right) a_{4}-a_{1}\left(a_{2}+a_{3}+a_{4}\right)+z_{1}+z_{2}, \\
& \delta=-\frac{\left(y \alpha+z_{1}\right)\left(y \beta+z_{2}\right)-a_{1} a_{2} a_{3} a_{4}}{y} .
\end{aligned}
$$

We need to choose how to define $z$, which we take to be specified by

$$
z_{1}=\frac{\left(y-a_{1}\right)\left(y-a_{2}\right)}{z}, \quad z_{2}=z\left(y-a_{3}\right)\left(y-a_{4}\right) .
$$

The theory regarding the existence and convergence of $Y_{0}(x)$ and $Y_{\infty}(x)$ for this case, where the asymptotics of the leading terms in the expansion of $A(x)$ around $x=0$ and $x=\infty$ are invertible, was determined very early by Birkhoff et al. [3]. In light of this, we solve for the first terms of $Y_{0}$ and $Y_{\infty}$ so that we may take the expansion to be

$$
Y_{0}(x)=\left(\left(\begin{array}{c}
\frac{w\left(\left(y \alpha+z_{1}\right) \kappa_{1}-\left(y \beta+z_{2}\right) \kappa_{2}+\lambda_{1}-\lambda_{2}\right)}{2 \kappa_{1}} \\
\delta
\end{array}\right.\right.
$$




$$
\begin{gathered}
\left.\left.\frac{w\left(\left(y \alpha+z_{1}\right) \kappa_{1}-\left(y \beta+z_{2}\right) \kappa_{2}-\lambda_{1}+\lambda_{2}\right)}{2 \kappa_{1}}\right)+O(x)\right)\left(\begin{array}{cc}
e_{q, \lambda_{1}}(x) & 0 \\
0 & e_{q, \lambda_{2}}(x)
\end{array}\right), \\
Y_{\infty}(x)=\left(I+\frac{1}{x}\left(\begin{array}{cc}
\frac{q(y+\alpha)}{q-1} & -\frac{q w}{q \kappa_{1}-\kappa_{2}} \\
\frac{q \gamma \kappa_{1}}{w\left(\kappa_{1}-q \kappa_{2}\right)} & \frac{q(y+\beta)}{q-1}
\end{array}\right)+O\left(\frac{1}{x^{2}}\right)\right)\left(\begin{array}{cc}
\frac{e_{q, \kappa_{1}}(x)}{\theta_{q}(x)^{2}} & 0 \\
0 & \frac{e_{q, \kappa_{2}}(x)}{\theta_{q}(x)^{2}}
\end{array}\right) .
\end{gathered}
$$

It is this parameterization in which we have a number of symmetries being introduced. We have the group of permutations on the $a_{i}$ generated by the elements

$$
\begin{aligned}
& s_{1}:\left\{\begin{array}{llll}
\kappa_{1} & \kappa_{2} & \lambda_{1} & \lambda_{2} \\
a_{1} & a_{2} & a_{3} & a_{4}
\end{array} ; w, y, z\right\} \rightarrow\left\{\begin{array}{cccc}
\kappa_{1} & \kappa_{2} & \lambda_{1} & \lambda_{2} \\
a_{2} & a_{1} & a_{3} & a_{4}
\end{array} ; w, y, z\right\}, \\
& s_{2}:\left\{\begin{array}{llll}
\kappa_{1} & \kappa_{2} & \lambda_{1} & \lambda_{2} \\
a_{1} & a_{2} & a_{3} & a_{4}
\end{array} ; w, y, z\right\} \rightarrow\left\{\begin{array}{cccc}
\kappa_{1} & \kappa_{2} & \lambda_{1} & \lambda_{2} \\
a_{1} & a_{3} & a_{2} & a_{4}
\end{array} ; w, y, z \frac{y-a_{3}}{y-a_{2}}\right\}, \\
& s_{3}:\left\{\begin{array}{llll}
\kappa_{1} & \kappa_{2} & \lambda_{1} & \lambda_{2} \\
a_{1} & a_{2} & a_{3} & a_{4}
\end{array} ; w, y, z\right\} \rightarrow\left\{\begin{array}{cccc}
\kappa_{1} & \kappa_{2} & \lambda_{1} & \lambda_{2} \\
a_{1} & a_{2} & a_{4} & a_{3}
\end{array} ; w, y, z\right\}
\end{aligned}
$$

which are all elementary Bäcklund transformations, as is the elementary transformation swapping the eigenvalues/asymptotic behavior at $x=0$, given by

$$
s_{4}:\left\{\begin{array}{cccc}
\kappa_{1} & \kappa_{2} & \lambda_{1} & \lambda_{2} \\
a_{1} & a_{2} & a_{3} & a_{4}
\end{array} ; w, y, z\right\} \rightarrow\left\{\begin{array}{cccc}
\kappa_{1} & \kappa_{2} & \lambda_{2} & \lambda_{1} \\
a_{1} & a_{2} & a_{3} & a_{4}
\end{array} ; w, y, z\right\} .
$$

To obtain the corresponding permutation of $\kappa_{1}$ and $\kappa_{2}$ we multiply $Y(x)$ on the left by

$$
R(x)=\left(\begin{array}{ll}
0 & 1 \\
1 & 0
\end{array}\right)
$$

in which the resulting transformation is most cleanly represented in terms of the parameterization of the linear problem, given as

$$
\begin{aligned}
s_{5}: & \left\{\begin{array}{llll}
\kappa_{1} & \kappa_{2} & \lambda_{1} & \lambda_{2} \\
a_{1} & a_{2} & a_{3} & a_{4}
\end{array} ; w, y, z\right\} \\
& \rightarrow\left\{\begin{array}{llll}
\kappa_{2} & \kappa_{1} & \lambda_{1} & \lambda_{2} \\
a_{1} & a_{2} & a_{3} & a_{4}
\end{array} ; \frac{\kappa_{1} \gamma}{w},-\frac{\delta}{\gamma}, z \frac{\left(\delta+\gamma a_{1}\right)\left(\delta+\gamma a_{2}\right)}{(y \gamma+\delta)(\beta \gamma+\delta)+\gamma^{2} z_{2}}\right\} .
\end{aligned}
$$

Since the operator, $T_{\kappa_{1}, \kappa_{2}, \lambda_{1}, \lambda_{2}}$, which is induced by $R_{\kappa_{1}, \kappa_{2}, \lambda_{1}, \lambda_{2}}(x)=x I$ is still a simple shift, if we include $T_{\kappa_{1}, \kappa_{2}, \lambda_{1}, \lambda_{2}}$ and the symmetries $s_{1}, \ldots, s_{5}$, we still need two non-trivial translations, $T_{a_{1}, \lambda_{1}}$ and $T_{\kappa_{1}, \lambda_{1}}$. These are specified by

$$
\begin{aligned}
T_{a_{1}, \lambda_{1}}: \tilde{w} & =w-\frac{w y\left(y-a_{1}\right) a_{1}\left(q \kappa_{1}-\kappa_{2}\right)}{a_{1}\left((z-1) y^{2}+\left(a_{1}-z\left(a_{3}+a_{4}\right)\right) y+z a_{3} a_{4}\right) \kappa_{2}+\left(y-a_{1}\right) \lambda_{2}}, \\
\tilde{y} & =\frac{a_{2} \kappa_{1}(w-\tilde{w})\left(a_{3} a_{4} \kappa_{2} \tilde{z}-q \lambda_{1}\right)}{\lambda_{1}\left(q \kappa_{1}(\tilde{w}+w(\tilde{z}-1))-\kappa_{2} \tilde{w} \tilde{z}\right)}, \\
\tilde{z} & =\frac{\kappa_{1}\left(a_{2}\left(q\left(w\left(y-a_{1}\right)+\tilde{w}\left(y(z-1)+a_{1}\right)\right) \kappa_{1}-w y z \kappa_{2}\right)+q z(w-\tilde{w}) \lambda_{1}\right)}{a_{2} \kappa_{1}\left(q w\left(y-a_{1}\right) \kappa_{1}+\left(\tilde{w}\left(y(z-1)+a_{1}\right)-w y z\right) \kappa_{2}\right)+z\left(q w \kappa_{1}-\tilde{w} \kappa_{2}\right) \lambda_{1}}, \\
T_{\kappa_{1}, \lambda_{1}}: \tilde{w} & =\frac{q^{2} w\left(\psi a_{1}+1\right)\left(\psi a_{2}+1\right) \kappa_{1}\left(q \kappa_{1}-z \kappa_{2}\right)(\tilde{z}-1)}{z \psi\left(\kappa_{2}-q \kappa_{1}\right)^{2} \tilde{z}}, \quad \tilde{y}=\frac{a_{3} a_{4} \kappa_{2} \tilde{z}-q \lambda_{1}}{q \psi \lambda_{1}-q \psi \lambda_{1} \tilde{z}}, \\
\tilde{z} z & =\frac{q^{2}\left(\psi a_{1}+1\right)\left(\psi a_{2}+1\right) \kappa_{1} \lambda_{1}}{\left(a_{3} \kappa_{2}+q \psi \lambda_{1}\right)\left(a_{4} \kappa_{2}+q \psi \lambda_{1}\right)},
\end{aligned}
$$


where

$$
\psi=\frac{y\left(z \kappa_{2}-q \kappa_{1}\right)}{q\left(a_{1} a_{2} \kappa_{1}-z \lambda_{1}\right)} .
$$

The remaining elementary Schlesinger transformations are obtained from the symmetries and the above shifts. This gives

$$
\mathbb{Z}^{7} \cong\left\langle T_{\kappa_{1}, \kappa_{2}, \lambda_{1}, \lambda_{2}}, T_{\kappa_{1}, \lambda_{1}}, T_{\kappa_{1}, \lambda_{2}}, T_{a_{1}, \lambda_{1}}, T_{a_{2}, \lambda_{1}}, T_{a_{3}, \lambda_{1}}, T_{a_{4}, \lambda_{1}}\right\rangle=G_{A} .
$$

We may identify the evolution of $q-\mathrm{P}_{\mathrm{VI}}$ with the connection preserving deformation

$$
q-\mathrm{P}_{\mathrm{VI}}:\left\{\begin{array}{cccc}
a_{1} & a_{2} & a_{3} & a_{4} \\
\kappa_{1} & \kappa_{2} & \lambda_{1} & \lambda_{2}
\end{array}: w, y, z\right\} \rightarrow\left\{\begin{array}{cccc}
q a_{1} & q a_{2} & a_{3} & a_{4} \\
\kappa_{1} & \kappa_{2} & q \lambda_{2} & q \lambda_{1}
\end{array}: \tilde{w}, \tilde{y}, \tilde{z}\right\}
$$

or in terms of Schlesinger transformations

$$
\begin{aligned}
q-\mathrm{P}_{\mathrm{VI}}=T_{a_{1}, \lambda_{1}, a_{2}, \lambda_{2}}: \tilde{w} & =\frac{q w \kappa_{1}(\tilde{z}-1)}{\kappa_{2} \tilde{z}-q \kappa_{1}}, \quad \tilde{y} y=-\frac{\left(a_{3} a_{4} \kappa_{2} \tilde{z}-q \lambda_{1}\right)\left(\lambda_{1} \tilde{z}-q a_{1} a_{2} \kappa_{1}\right)}{\lambda_{1}(\tilde{z}-1)\left(q \kappa_{1}-\kappa_{2} \tilde{z}\right)}, \\
\tilde{z} z & =\frac{q\left(y-a_{1}\right)\left(y-a_{2}\right) \kappa_{1}}{\left(y-a_{3}\right)\left(y-a_{4}\right) \kappa_{2}} .
\end{aligned}
$$

We make a the correspondence with (4.5) by letting $y=f$ and $z=g / b_{3}$, and the relations between parameters are given by

$$
a_{1}=b_{5}, \quad a_{2}=b_{6}, \quad a_{3}=b_{7}, \quad a_{4}=b_{8}, \quad \kappa_{1}=\frac{b_{4} \lambda_{1}}{b_{2} b_{7} b_{8}}, \quad \kappa_{1}=\frac{b_{3} \lambda_{1}}{b_{2} b_{7} b_{8}},
$$

which completes the correspondences. We remark that in the representation of (4.5) the transformation $b_{5} \rightarrow q b_{5}, b_{6} \rightarrow q b_{6}, b_{7} \rightarrow q b_{7}, b_{8} \rightarrow q b_{8}$ and $f \rightarrow q f$ is a trivial transformation and corresponds to $T_{a_{1}, a_{2}, a_{3}, a_{4}, \lambda_{1}^{2}, \lambda_{2}^{2}}$, which may also be regarded as trivial.

\section{Conclusion and discussion}

The above list comprises of $2 \times 2$ associated linear problems for $q$-Painlevé equations in which the associated linear problem is quadratic in $x$. The way in which we have increased the dimension of the underlying lattice of connection preserving deformations has been to successively increase the number of non-zero roots of the determinant. If we were to consider a natural extension of the $q-\mathrm{P}\left(A_{3}^{(1)}\right)$ case by adding a root making the determinant of order five in $x$ then we would necessarily obtain a matrix characteristically different from the Lax pair of Sakai [25] and Yamada [31]. Sakai adjoins two roots of the determinant while introducing a relation between $\kappa_{1}$ and $\kappa_{2}$ while Yamada [31] introduces four roots on top of the $q-\mathrm{P}\left(A_{3}^{(1)}\right)$ case and fixes the asymptotic behaviors at $x=0$ and $x=\infty$. This seems to skip the cases in which the determinant of $A$ is five. Of course, the above theory could be applied to those Lax pairs of Sakai and Yamada [25, 31], yet, the jump between the given characteristic data for the associated linear problems of $q-\mathrm{P}\left(A_{3}^{(1)}\right)$ and $q-\mathrm{P}\left(A_{2}^{(1)}\right)$ is intriguing, and worth exploring in greater depth.

Asides from the exceptional cases, where the symmetry of the underlying discrete Painlevé equations are of type $E_{6}^{(1)}, E_{7}^{(1)}$ or $E_{8}^{(1)}$, there are a few issues that need to be addressed. We have outlined a very natural lattice structure underlying the connection preserving deformations, however, it is unknown whether the full set of symmetries may be derived from the underlying connection preserving deformation setting. It seems that in order to fully address this, one would require a certain duality between roots of the determinant and the leading behavior at 0 and $\infty$. This could be explained from the point of view of rational matrices for example. We 
expect a framework which gives rise to a lift of the entire group of Bäcklund transformations to the level of Schlesinger transformations. This work, and the work of Yamada [31] may provide valuable insight into this.

We note the exceptional work of Noumi and Yamada in this case for a continuous analogue in their treatment of $\mathrm{P}_{\mathrm{VI}}$ [19]. They are able to extend the work of Jimbo and Miwa [11] for $\mathrm{P}_{\mathrm{VI}}$ in a manner that includes all the symmetries of $\mathrm{P}_{\mathrm{VI}}$. We remark however that the more geometric approach to Lax pairs explored by Yamada [32] gives us a greater insight into a possible fundamental link between the geometry and Lax integrability of the discrete Painlevé equations.

\section{References}

[1] Adams R.C., On the linear ordinary q-difference equation, Ann. of Math. 30 (1928), 195-205.

[2] Bellon M.P., Viallet C.-M., Algebraic entropy, Comm. Math. Phys. 204 (1999), 425-437, chao-dyn/9805006.

[3] Birkhoff G.D., General theory of linear difference equations, Trans. Amer. Math. Soc. 12 (1911), 243-284.

[4] Birkhoff G.D., The generalized Riemann problem for linear differential equations and the allied problems for linear difference and q-difference equations, Proc. Amer. Acad. 49 (1913), 512-568.

[5] Birkhoff G.D., Guenther P.E., Note on a canonical form for the linear q-difference system, Proc. Nat. Acad. Sci. USA 27 (1941), 218-222.

[6] Carmichael R.D., The general theory of linear q-difference equations, Amer. J. Math. 34 (1912), $147-168$.

[7] Di Vizio L., Ramis J.-P., Sauloy J., Zhang C., Équations aux q-différences, Gaz. Math. 96 (2003), 20-49.

[8] Fuchs R., Über lineare homogene Differentialgleichungen zweiter Ordnung mit drei im Endlichen gelegenen wesentlich singulären Stellen, Math. Ann. 63 (1907), 301-321.

[9] Fuchs R., Über lineare homogene Differentialgleichungen zweiter Ordnung mit drei im Endlichen gelegenen wesentlich singulären Stellen, Math. Ann. 70 (1911), 525-549.

[10] Gasper G., Rahman M., Basic hypergeometric series, Encyclopedia of Mathematics and its Applications, Vol. 35, Cambridge University Press, Cambridge, 1990.

[11] Jimbo M., Miwa T., Monodromy preserving deformation of linear ordinary differential equations with rational coefficients. II, Phys. D 2 (1981), 407-448.

[12] Jimbo M., Miwa T., Monodromy preserving deformation of linear ordinary differential equations with rational coefficients. III, Phys. D 4 (1981), 26-46.

[13] Jimbo M., Miwa T., Ueno K., Monodromy preserving deformation of linear ordinary differential equations with rational coefficients. I. General theory and $\tau$-function, Phys. D 2 (1981), 306-352.

[14] Jimbo M., Sakai H., A q-analog of the sixth Painlevé equation, Lett. Math. Phys. 38 (1996), 145-154, chao-dyn/9507010.

[15] Joshi N., Burtonclay D., Halburd R.G., Nonlinear nonautonomous discrete dynamical systems from a general discrete isomonodromy problem, Lett. Math. Phys. 26 (1992), 123-131.

[16] Le Caine J., The linear q-difference equation of the second order, Amer. J. Math. 65 (1943), 585-600.

[17] Murata M., Lax forms of the q-Painlevé equations, J. Phys. A: Math. Theor. 42 (2009), 115201, 17 pages, arXiv:0810.0058.

[18] Noumi M., An introduction to birational Weyl group actions, in Symmetric Functions 2001: Surveys of Developments and Perspectives, NATO Sci. Ser. II Math. Phys. Chem., Vol. 74, Kluwer Acad. Publ., Dordrecht, 2002, 179-222.

[19] Noumi M., Yamada Y., A new Lax pair for the sixth Painlevé equation associated with $\widehat{s o}(8)$, in Microlocal Analysis and Complex Fourier Analysis, World Sci. Publ., River Edge, NJ, 2002, 238-252, math-ph/0203029.

[20] Ormerod C.M., A study of the associated linear problem for $q-\mathrm{P}_{\mathrm{V}}$, arXiv:0911.5552.

[21] Papageorgiou V.G., Nijhoff F.W., Grammaticos B., Ramani A., Isomonodromic deformation problems for discrete analogues of Painlevé equations, Phys. Lett. A 164 (1992), 57-64.

[22] Ramani A., Grammaticos B., Hietarinta J., Discrete versions of the Painlevé equations, Phys. Rev. Lett. 67 (1991), 1829-1832. 
[23] Sakai H., Rational surfaces associated with affine root systems and geometry of the Painlevé equations, Comm. Math. Phys. 220 (2001), 165-229.

[24] Sakai H., A q-analog of the Garnier system, Funkcial. Ekvac. 48 (2005), 273-297.

[25] Sakai H., Lax form of the $q$-Painlevé equation associated with the $A_{2}^{(1)}$ surface, J. Phys. A: Math. Gen. 39 (2006), 12203-12210.

[26] Sauloy J., Galois theory of Fuchsian q-difference equations, Ann. Sci. École Norm. Sup. (4) 36 (2003), 925-968, math.QA/0210221.

[27] Shioda T., Takano K., On some Hamiltonian structures of Painlevé systems. I, Funkcial. Ekvac. 40 (1997), 271-291.

[28] Trjitzinsky W.J., Analytic theory of linear q-difference equations, Acta Math. 61 (1933), 1-38.

[29] van der Put M., Reversat M., Galois theory of q-difference equations, Ann. Fac. Sci. Toulouse Math. (6) 16 (2007), 665-718, math.QA/0507098.

[30] van der Put M., Singer M.F., Galois theory of difference equations, Lecture Notes in Mathematics, Vol. 1666, Springer-Verlag, Berlin, 1997.

[31] Yamada Y., Lax formalism for $q$-Painlevé equations with affine Weyl group symmetry of type $E_{n}^{(1)}$, Int. Math. Res. Not., to appear, arXiv:1004.1687.

[32] Yamada Y., A Lax formalism for the elliptic difference Painlevé equation, SIGMA 5 (2009), 042, 15 pages, arXiv:0811.1796. 